\title{
SOME REMARKS ON THE GENERAL THEOREM OF THE EXISTENCE OF ITERATIVE ROOTS OF HOMEOMORPHISMS WITH A RATIONAL ROTATION NUMBER
}

\author{
PAWEŁ SOLARZ ${ }^{1}$
}

\begin{abstract}
We show that the theorem proved in [8] generalises the previous results concerning orientationpreserving iterative roots of homeomorphisms of the circle with a rational rotation number (see [2], [6], [10] and [7]).
\end{abstract}

AMS (2000) Subject Classification. Primary 39B12; Secondary 26A18.

Keywords. iteration, iterative root, periodic point, rotation number, orientation-preserving mapping.

Résumé. Nous montrons que le théorème prouvé dans [8] généralise les résultats précédents concernant les racines itérées préservant l'orientation d'homéomorphismes du cercle avec un nombre de rotation rationnel (voir [2], [6], [10] et [7]).

Mots clefs. itération, racine itérée, point périodique, nombre de rotation, application préservant l'orientation.

A function $g: X \rightarrow X$, where $X \neq \emptyset$ is called an iterative root of a given function $f: X \rightarrow X$ if $g^{m}(x)=f(x)$ for $x \in X$, here $g^{m}$ denotes the $m$-th iterate of $g$ and $m \geq 2$, called the order of an iterative root, is an integer.

It seems that it was M. C. Zdun, who first started dealing with the problem of the existence of continuous and orientation-preserving iterative roots of an orientation-preserving homeomorphism of the circle, the homeomorphism with periodic or fixed points. Recall that $x \in X$ is a periodic point of order $n \in \mathbb{N}, n>1$ of $f$, if

$$
f^{n}(x)=x \text { and } f^{k}(x) \neq x \text { for } k \in\{1, \ldots, n-1\} .
$$

If $f(x)=x$ then $x$ is said to be a fixed point of $f$. The set of all periodic (fixed) points of $f$ will be denoted by Per $f($ Fix $f)$. In [9] Zdun solved the problem of the embedding of some homeomorphisms of the circle $S^{1}:=\{z \in \mathbb{C}:|z|=1\}$ in a continuous flow. Form these results one may conclude that if $F: S^{1} \rightarrow S^{1}$ is an orientation-preserving homeomorphism such that $S^{1} \neq \operatorname{Fix} F \neq \emptyset$, resp. Per $F=S^{1}$, then $F$ has infinitely many iterative roots of any order with fixed, resp. periodic points.

Three years later J.H. Mai in [5] gave some conditions for the existence of continuous iterative roots of $F$ with $S^{1} \neq \operatorname{Fix} F \neq \emptyset$ or $S^{1} \neq \operatorname{Per} F \neq \emptyset$.

In 2003 W. Jarczyk proved in [2] that if Fix $F=S^{1}$, i.e., $F$ is the identity function, then $F$ has infinitely many, depending on an arbitrary function, continuous and orientation-preserving iterative roots with periodic points. In the same year in [6] a similar result was proved for $F$ such that Per $F=S^{1}$.

In 2008 M.C. Zdun in [10] proved so called factorization theorem and as an application of it he gave the necessary and sufficient conditions for the existence of iterative roots with periodic points for $F$ such that $S^{1} \neq \operatorname{Fix} F \neq \emptyset$.

\footnotetext{
${ }^{1}$ Pedagogical University, Institute of Mathematics, ul. Podchorążych 2, PL-30-084 Krakó w, Poland; e-mail: psolarz@up.krakow.pl.
}

(c) EDP Sciences, SMAI 2012 
The mentioned Zdun's theorem was also used in [7] in the proof of Theorem 2 and for proving the general theorem in [8].

In this paper we show that the mentioned theorem from [8] generalises the results from [7], [10], [6] and [2]. Before that we recall some useful facts and definitions. From now on we set $\mathbb{Z}_{n}=\{0,1, \ldots, n-1\}$ and $\mathbb{Z}_{n}^{*}=\{1, \ldots, n-1\}$ for a suitable natural $n$.

Let $u, w \in S^{1}$ and $u \neq w$, then there exist $t_{1}, t_{2} \in \mathbb{R}$ such that $t_{1}<t_{2}<t_{1}+1$ and $u=e^{2 \pi \mathrm{i} t_{1}}$ and $w=e^{2 \pi \mathrm{i} t_{2}}$. Put

$$
\overrightarrow{(u, w)}:=\left\{e^{2 \pi \mathrm{i} t}, t \in\left(t_{1}, t_{2}\right)\right\}, \overrightarrow{[u, w]}:=\overrightarrow{(u, w)} \cup\{u, w\}, \overrightarrow{[u, w)}:=\overrightarrow{(u, w)} \cup\{u\} .
$$

These sets are called arcs.

For every homeomorphism $F: S^{1} \rightarrow S^{1}$ there exists a unique (up to a translation by an integer) homeomorphism $f: \mathbb{R} \rightarrow \mathbb{R}$ such that

$$
F\left(e^{2 \pi \mathrm{i} x}\right)=e^{2 \pi \mathrm{i} f(x)}
$$

and

$$
f(x+1)=f(x)+k
$$

for all $x \in \mathbb{R}$, where $k \in\{-1,1\}$. We call $F$ orientation-preserving if $k=1$, which is equivalent to the fact that $f$ is increasing.

Moreover, for every continuous function $G: I \rightarrow J$, where $I=\left\{e^{2 \pi \mathrm{i} t}, t \in[a, b]\right\}$ and $J=\left\{e^{2 \pi \mathrm{i} t}, t \in[c, d]\right\}$ there exists a unique continuous function $g:[a, b] \rightarrow[c, d]$ such that

$$
G\left(e^{2 \pi \mathrm{i} x}\right)=e^{2 \pi \mathrm{i} g(x)}, \quad x \in[a, b] .
$$

In this case we also call $g$ the lift of $G$ and we say that $G$ preserves the orientation if $g$ is strictly increasing.

For any orientation-preserving homeomorphism $F: S^{1} \rightarrow S^{1}$, the limit

$$
\alpha(F):=\lim _{n \rightarrow \infty} \frac{f^{n}(x)}{n}(\bmod 1), \quad x \in \mathbb{R}
$$

always exists and does not depend on the choice of $x$ and $f$. This number is called the rotation number of $F$ (see [1]). It is known that $\alpha(F)$ is a rational and positive number if and only if $F$ has a periodic point (see for example [1]). If $F: S^{1} \rightarrow S^{1}$ is an orientation-preserving homeomorphism such that $\alpha(F)=\frac{q}{n}$, where $q$, $n$ are positive integers with $0<q<n$ and $\operatorname{gcd}(q, n)=1$, then Per $F$ contains only periodic points of order $n$ (see [7], [4]). Moreover, there exists a unique number $p \in \mathbb{Z}_{n}^{*}$ satisfying $p q=1(\bmod n)$. This number will be called the characteristic number of $F$ and denoted char $F:=p$ (see $[10]$ ). If Fix $F \neq \emptyset$, then $\alpha(F)=0$ and we define $\operatorname{char} F:=1$.

Recall that if $G$ is an orientation-preserving iterative root of $F$, where $\alpha(F)=\frac{q}{n}, 0 \leq q<n$ and $\operatorname{gcd}(q, n)=1$, then there are an integer $l \geq 1$ and $q^{\prime} \in \mathbb{Z}_{n l}$ such that $\alpha(G)=\frac{q^{\prime}}{l n}$ and $\operatorname{gcd}\left(q^{\prime}, n l\right)=1$ (see for example Lemma 2 in [8]) and thus $G$ has periodic of fixed points of order $n l$.

In view of Theorem 5 (see [10]) every orientation-preserving homeomorphism $F: S^{1} \rightarrow S^{1}$ possessing periodic points of order $n$ is of the form

$$
F(z)= \begin{cases}T^{q}\left(F^{n}(z)\right), & z \in I_{0}=\overrightarrow{\left\langle z_{0}, F^{\text {char } F}\left(z_{0}\right)\right)} \\ T^{q}(z), & z \in S^{1} \backslash I_{0}\end{cases}
$$

where $z_{0} \in \operatorname{Per} F, q=n \alpha(F)$ and $T: S^{1} \rightarrow S^{1}$ is an orientation-preserving homeomorphism such that $T^{n}=\mathrm{id}_{S^{1}}$. The function $T=T_{z_{0}}(F)$ is unique up to a periodic point of $F$ and it is called the Babbage function of $F$ (see [10]). Of course if Per $F=S^{1}$, we have $T_{z_{0}}(F)=F^{\text {char } F}$. For an orientation-preserving homeomorphism such that Fix $F \neq \emptyset$ we may assume that the Babbage homeomorphism of $F$ is the identity function.

For the convenience we recall Theorem 2 from [8]. 


\section{THEOREM 1 .}

Let $m \geq 2$ and $l \geq 1$ be integers and let $F: S^{1} \rightarrow S^{1}$ be an orientation-preserving homeomorphism such that $\alpha(F)=\frac{q}{n}$, where $0 \leq q<n$ and $\operatorname{gcd}(q, n)=1$. $F$ has continuous and orientation-preserving iterative root of order $m$ with periodic points of order $l n$ if and only if the following conditions are fulfilled:

(i) $\frac{m}{l}=: m^{\prime} \in \mathbb{Z}$ and there is $q^{\prime} \in \mathbb{Z}_{n l}^{*}$ such that $\operatorname{gcd}\left(q^{\prime}, \ln \right)=1$ and $q^{\prime} m^{\prime}=q(\bmod n)$;

(ii) for some $z_{0} \in \operatorname{Per} F$ there $i s$ a partition of $I_{0}:=\overrightarrow{\left\langle z_{0}, F^{\text {char } F}\left(z_{0}\right)\right)}$ onto $l$ consecutive disjoint arcs $J_{0}, \ldots, J_{l-1}$ such that $F^{n}\left[J_{i}\right]=J_{i}, i \in \mathbb{Z}_{l}$ and if $l>1$, then there exist orientation-preserving homeomorphisms $V_{i}: J_{i} \rightarrow J_{i+1}, i \in \mathbb{Z}_{l-1}$ satisfying

$$
F_{\mid J_{i+1}}^{n}=V_{i} \circ F_{\mid J_{i}}^{n} \circ V_{i}^{-1}, \quad i \in \mathbb{Z}_{l-1}
$$

For any $z_{0} \in \operatorname{Per} F, m, l, q^{\prime}$, arcs $J_{0}, \ldots, J_{l-1}$ and homeomorphisms $V_{i}: J_{i} \rightarrow J_{i+1}, i \in \mathbb{Z}_{l-1}$ satisfying (1) the iterative root $G: S^{1} \rightarrow S^{1}$ of $F$ is of the form:

$$
G(z):= \begin{cases}V^{q^{\prime}}\left(G_{0}(z)\right), & z \in J_{0}, \\ V^{q^{\prime}}(z), & z \in S^{1} \backslash J_{0},\end{cases}
$$

where $G_{0}: J_{0} \rightarrow J_{0}$ is an orientation-preserving homeomorphism such that Fix $G_{0} \neq \emptyset$ and $G_{0}^{m^{\prime}}=F_{\mid J_{0}}^{n}$ and $V=\Psi^{\text {char } F}$ if $l=1$ or

$$
V(z):=\left\{\begin{array}{lc}
V_{i}(z), & z \in J_{i}, i \in \mathbb{Z}_{l-1}, \\
V_{l-1}(z):=\Psi^{\operatorname{char} F} \circ V_{0}^{-1} \circ \ldots \circ V_{l-2}^{-1}(z), & z \in J_{l-1}, \\
\Psi^{d \operatorname{char} F} \circ V_{i} \circ \Psi^{-d \operatorname{char} F}(z), & z \in F^{d \operatorname{char} F}\left[J_{i}\right], \\
& i \in \mathbb{Z}_{l}, \quad d \in \mathbb{Z}_{n}^{*}
\end{array}\right.
$$

if $l>1$, where $\Psi: S^{1} \rightarrow S^{1}$ is given by

$$
\Psi(z):=T^{q} \circ T^{d} \circ G_{i}^{\beta_{i, d}} \circ T^{-d}(z), \quad z \in F^{d \operatorname{char} F}\left[J_{i}\right], d \in \mathbb{Z}_{n}, i \in \mathbb{Z}_{l},
$$

where

$$
G_{j}:=V_{j} \circ G_{j-1} \circ V_{j}^{-1}, \quad j \in \mathbb{Z}_{l}^{*},
$$

$T=T_{z_{0}}(F)$ denotes the Babbage homeomorphism of $F$ and

$$
\beta_{i, d}:= \begin{cases}m^{\prime}-\left[\frac{m}{n l}\right]-1, & \text { if } d=0, i_{i}^{\prime} \leq m-\left[\frac{m}{n l}\right] n l-1, \\ m^{\prime}-\left[\frac{m}{n l}\right], & \text { if } d=0, i_{i}^{\prime}>m-\left[\frac{m}{n l}\right] n l-1, \\ -\left[\frac{m}{n l}\right]-1, & \text { if } d \in \mathbb{Z}_{n}^{*}, i_{i+d l}^{\prime} \leq m-\left[\frac{m}{n l}\right] n l-1, \\ -\left[\frac{m}{n l}\right], & \text { if } d \in \mathbb{Z}_{n}^{*}, i_{i+d l}^{\prime}>m-\left[\frac{m}{n l}\right] n l-1\end{cases}
$$

for $i \in \mathbb{Z}_{l}$ with $i_{k}^{\prime} \in \mathbb{Z}_{n l}$ uniquely determined by $\left(k+i_{k}^{\prime} q^{\prime}\right)(\bmod n l)=0$ for $k \in \mathbb{Z}_{n l}$.

Moreover, every orientation-preserving iterative root of order $m$ of $F$ with periodic points of order $n l$ (if exists) may be expressed by (2) - (6).

In order to obtain the first corollary, suppose that $F: S^{1} \rightarrow S^{1}$ is an orientation-preserving homeomorphism such that $S^{1} \neq \operatorname{Per} F \neq \emptyset, \alpha(F)=\frac{q}{n}$, where $\operatorname{gcd}(q, n)=1$ and that $l=1$. Then condition (i) of Theorem 1 takes the following form: there exists a $q^{\prime} \in \mathbb{Z}_{n}^{*}$ such that $\operatorname{gcd}\left(q^{\prime}, n\right)=1$ and $q^{\prime} m=q(\bmod n)$ which is equivalent to $\operatorname{gcd}(m, n)=1$ (see Remarks 2 and 3 in [7]). As $l=1$ we have $m^{\prime}=m, V=\Psi^{\text {char } F}$ and $J_{0}=I_{0}=\overrightarrow{\left\langle z_{0}, F^{\text {char } F}\left(z_{0}\right)\right)}$ for some $z_{0} \in \operatorname{Per} F$. Moreover, in (4) and (6) we have $i=0, d \in \mathbb{Z}_{n}$, thus $\beta_{i, d}=\beta_{0, d}=: \beta_{d}$ for $d \in \mathbb{Z}_{n}$. In addition, $i_{0}^{\prime}=0 \leq m-\left[\frac{m}{n}\right] n-1$. Therefore we get 


\section{Corollary 1.}

Let $F: S^{1} \rightarrow S^{1}$ be an orientation-preserving homeomorphism such that Per $F \neq S^{1}$ and $\alpha(F)=\frac{q}{n}$, where $\operatorname{gcd}(q, n)=1$. $F$ has orientation-preserving, continuous iterative root of order $m \geq 2$ with periodic point of order $n$ if and only if $\operatorname{gcd}(m, n)=1$.

For every such an $m$ and $z_{0} \in \operatorname{Per} F$ the iterative root $G: S^{1} \rightarrow S^{1}$ of $F$ is of the form

$$
G(z):= \begin{cases}\left(\Psi^{\operatorname{char} F}\right)^{q^{\prime}}\left(G_{0}(z)\right), & z \in I_{0}, \\ \left(\Psi^{\text {char } F}\right)^{q^{\prime}}(z), & z \in S^{1} \backslash I_{0},\end{cases}
$$

where $q^{\prime} \in \mathbb{Z}_{n}^{*}$ is such that $q^{\prime} m=q(\bmod n), G_{0}: I_{0} \rightarrow I_{0}$ is an orientation-preserving homeomorphism such that Fix $G_{0} \neq \emptyset, G_{0}^{m}=F_{\mid I_{0}}^{n}$ and $\Psi: S^{1} \rightarrow S^{1}$ is given by

$$
\Psi(z):=T^{q} \circ T^{d} \circ G_{0}^{\beta_{d}} \circ T^{-d}(z), \quad z \in F^{d \operatorname{char} F}\left[I_{0}\right], d \in \mathbb{Z}_{n},
$$

where $T=T_{z_{0}}(F)$ is the Babbage'a homeomorphism of $F$ and

$$
\beta_{d}:=\left\{\begin{array}{lll}
m-\left[\frac{m}{n}\right]-1, & d=0, & \\
-\left[\frac{m}{n}\right]-1, & d \in \mathbb{Z}_{n}^{*}, \quad i_{d}^{\prime} \leq m-\left[\frac{m}{n}\right] n-1, \\
-\left[\frac{m}{n}\right], & d \in \mathbb{Z}_{n}^{*}, \quad i_{d}^{\prime}>m-\left[\frac{m}{n}\right] n-1
\end{array}\right.
$$

with $i_{d}^{\prime} \in \mathbb{Z}_{n}$ such that $\left(d+i_{d}^{\prime} q^{\prime}\right)(\bmod n)=0$ for $d \in \mathbb{Z}_{n}$.

Moreover, every orientation-preserving iterative root of order $m$ of $F$ with periodic points of order $n$ (if exists) may be expressed by $(7)-(9)$.

This is a combination of Lemma 2 and Theorem 2 form [7].

Assume $l>1$ and let $F$ be such that $\emptyset \neq$ Fix $F \neq S^{1}$. For such a homeomorphism we have $\alpha(F)=0$, thus $q=0$ and $n=1$. By the definition, char $F=1$. Notice that for every $m$ such that $\frac{m}{l} \in \mathbb{Z}$ and every $q^{\prime} \in \mathbb{Z}_{l}^{*}$ such that $\operatorname{gcd}\left(q^{\prime}, l\right)=1$ we have $q^{\prime} m^{\prime}=0(\bmod 1)$, therefore the condition (i) of Theorem 1 is satisfied if $\frac{m}{l} \in \mathbb{Z}$. Moreover, in this case $\overrightarrow{\left\langle z_{0}, F^{\text {char } F}\left(z_{0}\right)\right)}=\overrightarrow{\left\langle z_{0}, F\left(z_{0}\right)\right\rangle}=S^{1}$ and $T_{z_{0}}(F)=\operatorname{Id}_{S^{1}}$ for any $z_{0} \in$ Fix $F$. Furthermore, in (4) and (6) we have $d=0, i \in \mathbb{Z}_{l}$ hence $i_{i}^{\prime} \geq 0>m-\left[\frac{m}{l}\right] l-1=-1$ for $i \in \mathbb{Z}_{l}$ and consequently, $\beta_{i, 0}=0$ for $i \in \mathbb{Z}_{l}$. Thus $\Psi=\operatorname{Id}_{S^{1}}$ and we get

Corollary 2.

Let $m, l \geq 2$ be integers. An orientation-preserving homeomorphism $F: S^{1} \rightarrow S^{1}$ with $\emptyset \neq$ Fix $F \neq S^{1}$ has a continuous and orientation-preserving iterative root of order $m$ with periodic point of order $l$ if and only if:

(i) $\frac{m}{l}=: m^{\prime} \in \mathbb{Z}$;

(ii) there is a partition of $S^{1}$ onto $l$ consecutive arcs $J_{0}, \ldots, J_{l-1}$ such that $F\left[J_{i}\right]=J_{i}, i \in \mathbb{Z}_{l}$ and there are orientation-preserving homeomorphisms $V_{i}: J_{i} \rightarrow J_{i+1}, i \in \mathbb{Z}_{l-2}$ such that

$$
F_{\mid J_{i+1}}=V_{i} \circ F_{\mid J_{i}} \circ V_{i}^{-1}, \quad i \in \mathbb{Z}_{l-1} .
$$

For every such a partition $\left\{J_{i}\right\}_{i \in \mathbb{Z}_{l}}$, numbers $m, l$, homeomorphisms $V_{i}, i \in \mathbb{Z}_{l-1}$ and each $0<q^{\prime}<l$ such that $\operatorname{gcd}\left(q^{\prime}, l\right)=1$ the function

$$
G(z):= \begin{cases}V^{q^{\prime}}\left(G_{0}(z)\right), & z \in J_{0}, \\ V^{q^{\prime}}(z), & z \in S^{1} \backslash J_{0},\end{cases}
$$

where $G_{0}: J_{0} \rightarrow J_{0}, G_{0}^{m^{\prime}}(z)=F(z)$ for $z \in J_{0}$ and Fix $G_{0} \neq \emptyset$ and

$$
V(z):= \begin{cases}V_{i}(z), & z \in J_{i}, i \in \mathbb{Z}_{l-1}, \\ V_{0}^{-1} \circ V_{1}^{-1} \circ \ldots \circ V_{l-2}^{-1}(z), & z \in J_{l-1},\end{cases}
$$


is the iterative root of $F$ such that Per $F \neq \emptyset$.

Moreover, every continuous and orientation-preserving iterative root of $F$ of order $m$ having periodic points of order $l$ is given by (10) and (11).

Taking $l=m$ in the above corollary we get Theorem 11 from [10].

Now turn to the case Per $F=S^{1}$ and $l>1$. As $F^{n}=\operatorname{Id}_{S^{1}}$ the condition (ii) of Theorem 2 is fulfilled for any partition of $I_{0}=\overrightarrow{\left\langle z_{0}, F^{\text {char } F}\left(z_{0}\right)\right)}$, where $z_{0} \in S^{1}$ and any orientation-preserving homeomorphisms $V_{i}: J_{i} \rightarrow J_{i+1}, i \in \mathbb{Z}_{l-1}$. In addition, $G_{0}: J_{0} \rightarrow J_{0}$ is the identity function as Fix $G_{0} \neq \emptyset$ and $G_{0}^{m^{\prime}}=F_{\mid J_{0}}^{n}=\operatorname{Id}_{J_{0}}$. This implies

$$
\Psi=T^{q}=\left(F^{\text {char } F}\right)^{q}=F
$$

as $T=T_{z_{0}}(F)=F^{\text {char } F}$. Hence

Corollary 3.

Let $m, l \geq 2$ be integers, $F: S^{1} \rightarrow S^{1}$ be an orientation-preserving homeomorphism such that Per $F=S^{1}$ and $\alpha(F)=\frac{q}{n}$, where $0<q<n$ and $\operatorname{gcd}(q, n)=1$. F has a continuous and orientation-preserving iterative root of order $m$ with periodic point of order $n l$ if and only if:

(i) $\frac{m}{l}=: m^{\prime} \in \mathbb{Z}$ and there is $q^{\prime} \in \mathbb{Z}_{\text {ln }}^{*}$ such that $\operatorname{gcd}\left(q^{\prime}, \ln \right)=1$ and $q^{\prime} m^{\prime}=q(\bmod n)$.

For every $m, q^{\prime}, l$ satisfying (i) every partition $\left\{J_{i}\right\}_{i \in \mathbb{Z}_{l}}$ of $I_{0}$ and every orientation-preserving homeomorphisms $V_{i}: J_{i} \rightarrow J_{i+1}, i \in \mathbb{Z}_{l-1}$ the iterative root $G: S^{1} \rightarrow S^{1}$ of $F$ is of the form:

$$
G(z):=V^{q^{\prime}}(z), \quad z \in S^{1}
$$

where

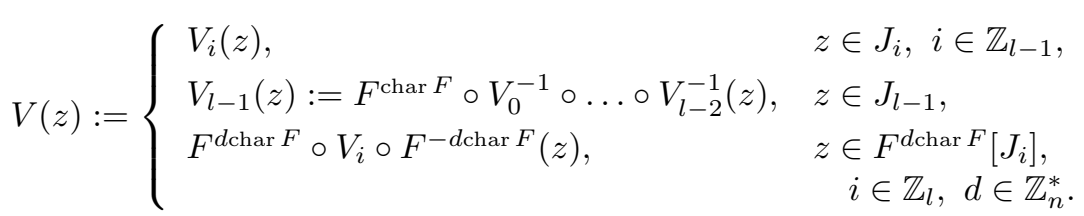

Moreover, every continuous and orientation-preserving iterative root of $F$ of order $m$ having periodic points of order $n l$ is given by (12) and (13).

This is slightly modified Theorem 2 from [6].

Assume that Per $F=S^{1}$ and $l=1$. Similarly as in Corollary 1 the condition (i) is equivalent to $\operatorname{gcd}(m, n)=1$ and the condition (ii) is not the case. Moreover, $\Psi=F$ and $V=F^{\text {char } F}$. Hence

\section{Corollary 4.}

Let $m \geq 2$ be integer, $F: S^{1} \rightarrow S^{1}$ be an orientation-preserving homeomorphism such that Per $F=S^{1}$ and $\alpha(F)=\frac{q}{n}$, where $0<q<n$ and $\operatorname{gcd}(q, n)=1$. F has a continuous and orientation-preserving iterative root of order $m$ with periodic point of order $n$ if and only if $\operatorname{gcd}(m, n)=1$. For every $m \geq 2$ and every $q^{\prime} \in \mathbb{Z}_{n}^{*}$ such that $\operatorname{gcd}\left(q^{\prime}, n\right)=1$ and $q^{\prime} m=q(\bmod n)$ the iterative root $G: S^{1} \rightarrow S^{1}$ of $F$ is of the form:

$$
G(z)=\left(F^{\text {char } F}\right)^{q^{\prime}}(z), \quad z \in S^{1}
$$

This is Corollary 4 from [6]. In [6] this result was obtained independently on Theorem 2.

Finally suppose that $l>1$ and Fix $F=S^{1}$, i.e., $F=\operatorname{Id}_{S^{1}}$. Since $q=0, n=1$, we have char $F=1, I_{0}=S^{1}$ and $T=T_{z_{0}}(F)=\operatorname{Id}_{S^{1}}$ for any $z_{0} \in S^{1}$. Notice that if $\frac{m}{l} \in \mathbb{Z}$ then every $q^{\prime} \in \mathbb{Z}_{l}^{*} \operatorname{such} \operatorname{that} \operatorname{gcd}\left(q^{\prime}, l\right)=1$ satisfies $m^{\prime} q^{\prime}=0(\bmod 1)$. The condition (ii) is obviously fulfilled for any partition $\left\{J_{i}\right\}_{i \in \mathbb{Z}_{l}}$ of $S^{1}$ and any orientation-preserving homeomorphisms $V_{i}: J_{i} \rightarrow J_{i+1}, i \in \mathbb{Z}_{l-1}$. Moreover as $G_{0}=\operatorname{Id}_{J_{0}}$ we have $\Psi=\operatorname{Id}_{S^{1}}$. Therefore from Theorem 1 it follows the Jarczyk's theorem from [2]. 


\section{COROllary 5.}

The function $F=\operatorname{Id}_{S^{1}}$ has continuous and orientation-preserving iterative roots of order $m \geq 2$ with periodic points of order $l>1$ if and only if $\frac{m}{l} \in \mathbb{Z}$.

For every such numbers $m, l$, every partition $\left\{J_{i}\right\}_{i \in \mathbb{Z}_{l}}$ of $S^{1}$ and every orientation-preserving homeomorphisms $V_{i}: J_{i} \rightarrow J_{i+1}, i \in \mathbb{Z}_{l-1}$, the function

$$
G(z):=V^{q^{\prime}}(z), \quad z \in S^{1}
$$

where $q^{\prime} \in \mathbb{Z}_{l}^{*}$ is such that $\operatorname{gcd}\left(q^{\prime}, l\right)=1$ and

$$
V(z):= \begin{cases}V_{i}(z), & z \in J_{i}, i \in \mathbb{Z}_{l-1}, \\ V_{0}^{-1} \circ V_{1}^{-1} \circ \ldots \circ V_{l-2}^{-1}(z), & z \in J_{l-1}\end{cases}
$$

is the orientation-preserving continuous iterative root of $\operatorname{Id}_{S^{1}}$ of order $l$. Moreover, every continuous and orientation-preserving iterative root of $\mathrm{Id}_{S^{1}}$ of order $m$ having periodic points of order $l$ is given by (14) and (15).

\section{REFERENCES}

[1] I. P. Cornfeld, S. V. Fomin, Y. G. Sinai, Ergodic theory, Grundlehren Math. Wiss. 245, Spirnger-Verlag, Berlin, Heidelberg, New York, 1982

[2] W. Jarczyk, Babbage equation on the circle, Publ. Math. Debrecen 63 (2003), no. 3, 389-400.

[3] M. Kuczma, B. Choczewski, R. Ger, Iterative functional equations, Encyclopaedia Math. Appl. 32, Cambridge Univ. Press, Cambridge, 1990.

[4] W. de Melo, S. van Strien, One-dimensional dynamics, Ergeb. Math. Grenzgeb. (3), Band 25, Springer-Verlag, Berlin 1993.

[5] J. H. Mai, Conditions for the existence of $n$th iterative roots of self-homeomorphisms of the circle, (Chinese), Acta Math. Sinica 30 (1987), no.2, 280-283.

[6] P. Solarz, On some iterative roots, Publ. Math. Debrecen 63 (2003), no. 4, 677-692.

[7] P. Solarz, A note on some iterative roots, Ann. Univ. Paed. Cracov. Stud. Math. 8 (2009), 57-66.

[8] P. Solarz, General theorem for the existence of iterative roots of homeomorphisms with periodic points, J. Math. Anal. Appl. 394 (2012), 391-399.

[9] M. C. Zdun, On embedding of homeomorphisms of the circle in continuous flow, Iteration theory and its functional equations, (Proceedings, Schloss Hofen, 1984), Lecture Notes in Mathematics 1163, Springer-Verlag, Heidelberg, New York, 1985, $218-231$.

[10] M. C. Zdun, On a factorization of homeomorphisms of the circle possessing periodic points, J. Math. Anal. Appl. 342 (2008), 340-348. 